\title{
Comparative study: quality of life between Assiut university hospital \& Umulj hospital for patients with end stage renal disease undergoing hemodialysis
}

\author{
Nagwa M. Ahmed, Sahra Z. Azer \& Sahar A. Abd-El mohsen. \\ Lecturer of Adult Nursing Dept., Faculty of Nursing, Assiut University, Assiut
}

\begin{abstract}
The present study aimed to; determine the quality of life of hemodialysis patients and to compare the quality of life of hemodialysis patients at Assiut University hospital and at Umulj hospital, Tabuk. Data were collected from the hemodialysis center at Assiut University hospital and at Umulj hospital, Tabuk. The study was conducted on 80 adult patients, having the following criteria; age between 18 - 65 years, both male and female patients with end stage renal disease on hemodialysis, 40 patients from each dialysis center. Data were collected through the following tools; Tool (I): Sociodemographic data sheet; including age, gender, marital status, educational level, occupation, residence, family number, duration of hemodialysis....etc. and Tool (II): World Health Organization Quality Of Life (WHOQOL); including 5 domains which are physical, social, psychological, environment, and perceived QOL. Results of this study; near half of the studied sample their age has ranged from 50 - 65 years in both groups, and more than half of the studied samples in both hospitals were having a moderate score in their quality of life. Conclusion; there was no statistically significant difference between both studied samples regarding their quality of life level, also a statistically significant relation was found between the studied groups regarding their quality of life and both patient's gender and occupation. The study recommended that; providing counseling for patient with end stage renal disease on hemodialysis will play an important role in improving the QOL of those patients.
\end{abstract}

Key words: quality of life, end stage renal disease \& hemodialysis.

\section{Introduction}

Chronic renal failure, or end - stage renal disease (ESRD) is a progressive, irreversible deterioration in renal function in which the body's ability to maintain metabolic and fluid and electrolyte balance fails, resulting in uremia or azotemia (Brunner, 2010).

End-stage renal disease (ESRD) is a life-threatening condition and survival can be maintained only with renal replacement therapy. Treatment options for the disease often involve either long-term dialysis or kidney transplantation. Furthermore, the complications of ESRD, its treatment and co-existing diseases have been found to have a significant impact on the physical health of patients. It is well documented that the health status of the hemodialysis population is worse than that of the general healthy population (Tel, 2011).

Hemodialysis (HD) is a life-saving treatment for the patients with end-stage renal disease (ESRD) requiring renal replacement therapy (Kusleikaite, Bumblyte, Kuzminskis, \& Vaiciuniene, 2010). The health-related quality of life (HRQOL) is lessened in patients with ESRD as expected in those with chronic illness (Turkmen, Yazici, Solak, Guney, Altintepe, \& Yeksan, 2011), because these patients have many fears and various necessities (De Santo, Perna, EI Matri, \& Cirillo, 2012).

The effectiveness of health care and development of health policies are often determined by HRQOL assessments (Braga, Peixoto, Gomes, de Assis Acurcio, Andrade, Cherchiglia, 2011). HRQOL is also an important predictor of HD patient's outcomes that should be frequently assessed (GerminPetrovic, Mesaros-Devcic, Lesac, Mandic, Soldatic, \& Vezmar, 2011).

The HRQOL measurement indicates the impact of illness on the patient's physical, mental, and social performance (Kusleikaite, et al 2010). Since hemodialysis is an expensive treatment modality for chronic renal failure patients, it is very essential to assess the outcome of therapy in terms of quality of life (Abraham \& Ramachandran, 2012).

QOL is used to evaluate the general well being of individuals and societies. It may vary according to the patient as well as the disease condition. The World Health Organization defines Quality of life as "an individual's perception of their position in life in the context of the culture and value systems in which they live and in relation to their goals, expectations, standards and concerns. It is a broad ranging concept affected in a complex way by the person's physical health, psychological state, personal beliefs, social relationships and their relationship to salient features of their environment"(Oort, 2005) 


\section{Aim of the study}

To determine the quality of life of end-stage renal disease patients undergoing hemodialysis.

To compare the quality of life of end-stage renal disease patients undergoing hemodialysis at Assiut University hospital and Umulj hospital, Tabuk.

\section{Research question}

Is there a difference in the quality of life of patients with end stage renal disease undergoing hemodialysis in Assiut University hospital and Umulj hospital?

\section{Subjects and method}

\section{Study design}

Descriptive study design was utilized in this study.

\section{Setting:}

The study was conducted in the hemodialysis unit at Assiut university hospital and Umulj hospital, Tabuk.

\section{Sample}

A convenience sample of 40 adult patients attending the hemodialysis unit, at Assiut University Hospital, and 40 other patients undergoing hemodialysis in Umulj hospital, Tabuk at Kingdom Umulj both male and female, and their age from 18 to 65 years were included in this study.

\section{Tools of data collection:}

\section{Data was collected through use of the following} tools:

\section{Tool (I): Sociodemographic data sheet:}

It was developed by the researchers including age, gender, marital status, educational level, occupation, residence, family number, duration of hemodialysis, number of hemodialysis sessions, and number of hemodialysis hours per week

Tool (II): World Health Organization Quality Of Life (Whoqol):

The (Whoqol): developed by WHO (2004). (King \& Hinds) The tool contains 54 questions over 5 broad domains of QOL within which 18 facets are covered to determine the quality of life. These 5 domains include physical, social, psychological, environment, and perceived QOL. Within each domain, several sub domains (facets) of QOL Summarized that particular domain of QOL. The 5 main domains are:

1-Physical health including: Activities of daily living, pain and discomfort, energy and fatigue, sleep and rest, work capacity, mobility and dependence on medication.

2-Psychological including: Negative feeling, positive feeling, self esteem, spiritual, religion and personal beliefs.

3-Social relationships including: Personal relationships, social support and sexual activity.

4-Environment including: Physical environment, safety and security, health and social care.

5-Perceived quality of life.

\section{6-Distribution of the scores in QOL tool}

\begin{tabular}{|l|c|c|}
\hline Domain & Items & Scord \\
\hline Physical & 7 & 63 \\
Psychological & 4 & 36 \\
Social & 3 & 27 \\
Environmental & 3 & 27 \\
Perceived QOL & 1 & 9 \\
\hline Total score QOL & 18 & 162 \\
\hline
\end{tabular}

7-The scoring for these variables, a 3-point Lickert scale on tables was adopted for the answer low $\mathrm{QOL}=0-3$, moderate $\mathrm{QOL}=4-6$, and high $\mathrm{QOL}=7-9$ Quality of life scoring system

The tools used for data collection in this study were all previously validated the initial reliability analyses showed a correlation coefficient of more than 0.4-0.8. The validity of the tool ranged from $\mathrm{r}=0.244$ and 0.676 by (kuyken et al., 1994), This Arabic version (translated by Professor Mohamed Abraham kamel, faculty of medicine, Alexandria, Assiut) was used in this study tool in Assiut by Mahmud, 2001 and modified by the researcher. The content validity of this tool was checked by expert professors in fields of medicine and nursing and correction was carried out accordingly for scoring for these variables, 3-points Lickert scale was adopted for the answer of each questions, which ranged from never (1)= low QOL, $(2)=$ moderate $\mathrm{QOL}, \operatorname{much}(3)=$ high $\mathrm{QOL}$. For pain and discomfort, the range in the sheets was from never (1), moderate (2), much (3). Each facet comprised 3 questions, i.e., the highest score for every facet was $0-9$. 


\section{Results}

Table (1): Distribution of the biosciodemographic hemodialysis patient characteristics in Assiut university hospital and Umulj hospital $(n=80)$.

\begin{tabular}{|c|c|c|c|c|}
\hline \multirow{3}{*}{ Variable } & \multicolumn{4}{|c|}{ Country } \\
\hline & \multicolumn{2}{|c|}{ Assiut $(n=40)$} & \multicolumn{2}{|c|}{ Umulj $(n=40)$} \\
\hline & No. & $\%$ & No. & $\%$ \\
\hline \multicolumn{5}{|l|}{ Age groups } \\
\hline $18-29$ years & 6 & 15.0 & 9 & 22.5 \\
\hline $30-39$ years & 11 & 27.5 & 2 & 5.0 \\
\hline $40-49$ years & 10 & 25.0 & 10 & 25.0 \\
\hline $50-65$ years & 13 & 32.5 & 19 & 47.5 \\
\hline Mean \pm SD & \multicolumn{2}{|c|}{$42.0 \pm 11.9$} & \multicolumn{2}{|c|}{$44.4 \pm 14.6$} \\
\hline \multicolumn{5}{|l|}{ Gender } \\
\hline Male & 21 & 52.5 & 21 & 52.5 \\
\hline Female & 19 & 47.5 & 19 & 47.5 \\
\hline \multicolumn{5}{|l|}{ Marital status } \\
\hline Single & 5 & 12.5 & 9 & 22.5 \\
\hline Married & 32 & 80.0 & 20 & 50.0 \\
\hline Divorced & 0 & 0.0 & 5 & 12.5 \\
\hline Widowed & 3 & 7.5 & 6 & 15.0 \\
\hline \multicolumn{5}{|l|}{ Education level } \\
\hline Illiterate & 13 & 32.5 & 14 & 35.0 \\
\hline Reading and writing & 5 & 12.5 & 10 & 25.0 \\
\hline Basic education & 4 & 10.0 & 12 & 30.0 \\
\hline Secondary school & 15 & 37.5 & 0 & 0.0 \\
\hline University & 3 & 7.5 & 4 & 10.0 \\
\hline \multicolumn{5}{|l|}{ Occupation } \\
\hline Not working & 15 & 37.5 & 19 & 47.5 \\
\hline Worker & 2 & 5.0 & 3 & 7.5 \\
\hline Employee & 6 & 15.0 & 6 & 15.0 \\
\hline Student & 2 & 5.0 & 0 & 0.0 \\
\hline House wife & 14 & 35.0 & 12 & 30.0 \\
\hline Others & 1 & 2.5 & 0 & 0.0 \\
\hline \multicolumn{5}{|l|}{ Residence } \\
\hline City & 16 & 40.0 & 33 & 82.5 \\
\hline Rural & 24 & 60.0 & 7 & 17.5 \\
\hline \multicolumn{5}{|l|}{ Family number } \\
\hline $1-3$ person & 7 & 17.5 & 6 & 15.0 \\
\hline $4-6$ person & 20 & 50.0 & 11 & 27.5 \\
\hline $7+$ person & 13 & 32.5 & 23 & 57.5 \\
\hline Range & \multicolumn{2}{|c|}{$2-9$} & \multicolumn{2}{|c|}{$1-18$} \\
\hline Mean \pm SD & \multicolumn{2}{|c|}{$5.6 \pm 2.1$} & \multicolumn{2}{|c|}{$7.8 \pm 3.9$} \\
\hline \multicolumn{5}{|l|}{ Duration of hemodialysis } \\
\hline 6 months $<1$ year & 4 & 10.0 & 7 & 17.5 \\
\hline 1 year $<3$ years & 10 & 25.0 & 5 & 12.5 \\
\hline 3 years \& more & 26 & 65.0 & 28 & 70.0 \\
\hline \multicolumn{5}{|c|}{ Number of hemodialysis sessions } \\
\hline Two & 1 & 2.5 & 0 & 0.0 \\
\hline Three or more & 39 & 97.5 & 40 & 100.0 \\
\hline
\end{tabular}




\begin{tabular}{|l|c|c|c|c|}
\hline \multirow{2}{*}{ Variable } & \multicolumn{4}{|c|}{ Country } \\
\cline { 2 - 5 } & \multicolumn{2}{|c|}{ Assiut (n=40) } & \multicolumn{2}{c|}{ Umulj (n=40) } \\
\cline { 2 - 5 } & No. & \% & No. & \% \\
\hline Number of hemodialysis session hours /week & 39 & 97.5 & 12 & 30.0 \\
\hline Four & 1 & 2.5 & 28 & 70.0 \\
\hline Five & \multicolumn{3}{|c|}{} \\
\hline
\end{tabular}

Table (2): Distribution of the studied sample as regards to physical domain $(\mathrm{n}=80)$.

\begin{tabular}{|c|c|c|c|c|c|c|c|c|c|c|c|c|c|c|}
\hline \multirow{3}{*}{$\begin{array}{l}\text { Physical } \\
\text { domain }\end{array}$} & \multicolumn{7}{|c|}{ Assiut $(n=40)$} & \multicolumn{7}{|c|}{ Umulj (n=40) } \\
\hline & \multicolumn{2}{|c|}{ Low } & \multicolumn{2}{|c|}{ Moderate } & \multicolumn{2}{|c|}{ High } & \multirow{2}{*}{$\operatorname{Mean} \pm$ SD } & \multicolumn{2}{|c|}{ Low } & \multicolumn{2}{|c|}{ Moderate } & \multicolumn{2}{|c|}{ High } & \multirow[t]{2}{*}{$\begin{array}{c}\text { Mean } \pm \\
\text { SD } \\
\end{array}$} \\
\hline & No & $\%$ & No & $\%$ & No & $\%$ & & No & $\%$ & No & $\%$ & No & $\%$ & \\
\hline \multicolumn{15}{|c|}{ 1- Doing daily activities: } \\
\hline $\begin{array}{l}- \\
\text { you able to do daily } \\
\text { activities? }\end{array}$ & 10 & $\begin{array}{c}25 . \\
0\end{array}$ & 23 & $\begin{array}{c}5 \\
7 . \\
5\end{array}$ & 7 & 17.5 & $1.9 \pm 0.7$ & 6 & $\begin{array}{l}15 \\
.0\end{array}$ & 31 & 77.5 & 3 & 7.5 & $1.9 \pm 0.5$ \\
\hline $\begin{array}{l}\text { How far } \\
\text { do you feeling } \\
\text { difficulty in doing } \\
\text { your usual activities? }\end{array}$ & 16 & $\begin{array}{c}40 . \\
0\end{array}$ & 12 & $\begin{array}{l}3 \\
0 . \\
0\end{array}$ & 12 & 30.0 & $1.9 \pm 0.8$ & 8 & $\begin{array}{c}20 \\
.0\end{array}$ & 29 & 72.5 & 3 & 7.5 & $1.9 \pm 0.5$ \\
\hline $\begin{array}{l}\text { How far } \\
\text { do you upset failure } \\
\text { in doing activities? }\end{array}$ & 12 & $\begin{array}{c}30 . \\
0\end{array}$ & 16 & $\begin{array}{l}4 \\
0 . \\
0\end{array}$ & 12 & 30.0 & $2 \pm 0.8$ & 10 & $\begin{array}{c}25 \\
.0\end{array}$ & 25 & 62.5 & 5 & 12.5 & $1.9 \pm 0.6$ \\
\hline \multicolumn{15}{|c|}{ 2- Pain and discomfort: } \\
\hline $\begin{array}{l}\text { How } \\
\text { often do you suffer } \\
\text { from Pains? }\end{array}$ & 0 & 0.0 & 21 & $\begin{array}{c}5 \\
2 . \\
5\end{array}$ & 19 & 47.5 & $2.5 \pm 0.5$ & 5 & $\begin{array}{l}12 \\
.5\end{array}$ & 25 & 62.5 & 10 & 25.0 & $2.1 \pm 0.6$ \\
\hline \begin{tabular}{l}
\multicolumn{1}{c}{ Do you } \\
worry about Pains or \\
discomfort?
\end{tabular} & 0 & 0.0 & 12 & $\begin{array}{l}3 \\
0 . \\
0\end{array}$ & 28 & 70.0 & $2.7 \pm 0.5$ & 0 & $\begin{array}{l}0 . \\
0\end{array}$ & 28 & 70.0 & 12 & 30.0 & $2.3 \pm 0.5$ \\
\hline $\begin{array}{l}- \text { How } \\
\text { much don`t you do } \\
\text { your works when } \\
\text { you feel pain? }\end{array}$ & 29 & $\begin{array}{c}72 . \\
5\end{array}$ & 11 & $\begin{array}{l}2 \\
7 . \\
5\end{array}$ & 0 & 0.0 & $1.3 \pm 0.5$ & 10 & $\begin{array}{c}25 \\
.0\end{array}$ & 29 & 72.5 & 1 & 2.5 & $1.8 \pm 0.5$ \\
\hline \multicolumn{15}{|l|}{ 3- Energy and fatigue: } \\
\hline $\begin{array}{l}\text { Do you } \\
\text { have enough energy } \\
\text { for daily activities? }\end{array}$ & 7 & $\begin{array}{c}17 . \\
5\end{array}$ & 27 & $\begin{array}{c}6 \\
7 . \\
5 \\
\end{array}$ & 6 & 15.0 & $2 \pm 0.6$ & 12 & $\begin{array}{c}30 \\
.0\end{array}$ & 24 & 60.0 & 4 & 10.0 & $1.8 \pm 0.6$ \\
\hline $\begin{array}{l}\text { How far do you } \\
\text { satisfy/pleased with } \\
\text { your energy? }\end{array}$ & 19 & $\begin{array}{c}47 . \\
5\end{array}$ & 14 & $\begin{array}{c}3 \\
5 . \\
0 \\
\end{array}$ & 7 & 17.5 & $1.7 \pm 0.8$ & 4 & $\begin{array}{l}10 \\
.0\end{array}$ & 32 & 80.0 & 4 & 10.0 & $2 \pm 0.5$ \\
\hline $\begin{array}{l}\text { How far do you upset } \\
\text { your pain? }\end{array}$ & 20 & $\begin{array}{c}50 . \\
0\end{array}$ & 9 & $\begin{array}{c}2 \\
2 . \\
5\end{array}$ & 11 & 27.5 & $1.8 \pm 0.9$ & 17 & $\begin{array}{l}42 \\
.5\end{array}$ & 21 & 52.5 & 2 & 5.0 & $1.6 \pm 0.6$ \\
\hline \multicolumn{15}{|l|}{ 4- Sleep and rest } \\
\hline Do you sleep well? & 5 & $\begin{array}{c}12 . \\
5\end{array}$ & 31 & $\begin{array}{c}7 \\
7 . \\
5\end{array}$ & 4 & 10.0 & $2 \pm 0.5$ & 2 & $\begin{array}{l}5 . \\
0\end{array}$ & 30 & 75.0 & 8 & 20.0 & $2.2 \pm 0.5$ \\
\hline $\begin{array}{l}\text { Do you suffer from } \\
\text { difficulty sleeping? }\end{array}$ & 7 & $\begin{array}{c}17 . \\
5\end{array}$ & 25 & $\begin{array}{c}6 \\
2 . \\
5\end{array}$ & 8 & 20.0 & $2 \pm 0.6$ & 14 & $\begin{array}{l}35 \\
.0\end{array}$ & 24 & 60.0 & 2 & 5.0 & $1.7 \pm 0.6$ \\
\hline $\begin{array}{l}\text { How far do you } \\
\text { worry about sleeping } \\
\text { problems? }\end{array}$ & 18 & $\begin{array}{c}45 . \\
0\end{array}$ & 15 & $\begin{array}{l}3 \\
7 . \\
5 \\
\end{array}$ & 7 & 17.5 & $1.7 \pm 0.8$ & 16 & $\begin{array}{l}40 \\
.0\end{array}$ & 21 & 52.5 & 3 & 7.5 & $1.7 \pm 0.6$ \\
\hline \multicolumn{15}{|c|}{ 5- The capability to work } \\
\hline Do you able to work? & 19 & 47. & 16 & 4 & 5 & 12.5 & $1.7 \pm 0.7$ & 21 & 52 & 17 & 42.5 & 2 & 5.0 & $1.5 \pm 0.6$ \\
\hline
\end{tabular}




\begin{tabular}{|c|c|c|c|c|c|c|c|c|c|c|c|c|c|c|}
\hline \multirow{3}{*}{$\begin{array}{l}\text { Physical } \\
\text { domain }\end{array}$} & \multicolumn{7}{|c|}{ Assiut $(n=40)$} & \multicolumn{7}{|c|}{ Umulj (n=40) } \\
\hline & \multicolumn{2}{|c|}{ Low } & \multicolumn{2}{|c|}{ Moderate } & \multicolumn{2}{|c|}{ High } & \multirow{2}{*}{ Mean \pm SD } & \multicolumn{2}{|c|}{ Low } & \multicolumn{2}{|c|}{ Moderate } & \multicolumn{2}{|c|}{ High } & \multirow[t]{2}{*}{$\begin{array}{c}\text { Mean } \pm \\
\text { SD }\end{array}$} \\
\hline & No & $\%$ & No & $\%$ & No & $\%$ & & No & $\%$ & No & $\%$ & No & $\%$ & \\
\hline & & 5 & & $\begin{array}{c}0 . \\
0\end{array}$ & & & & & .5 & & & & & \\
\hline $\begin{array}{l}\text { Do you feel } \\
\text { capability in doing } \\
\text { duties? }\end{array}$ & 17 & $\begin{array}{c}42 . \\
5\end{array}$ & 17 & $\begin{array}{l}4 \\
2 . \\
5\end{array}$ & 6 & 15.0 & $1.7 \pm 0.7$ & 7 & $\begin{array}{r}17 \\
.5\end{array}$ & 28 & 70.0 & 5 & 12.5 & $2 \pm 0.6$ \\
\hline $\begin{array}{l}\text { How far do you } \\
\text { satisfy your } \\
\text { capability to work? }\end{array}$ & 20 & $\begin{array}{c}50 . \\
0\end{array}$ & 15 & $\begin{array}{l}3 \\
7 . \\
5 \\
\end{array}$ & 5 & 12.5 & $1.6 \pm 0.7$ & 11 & $\begin{array}{r}27 \\
.5\end{array}$ & 25 & 62.5 & 4 & 10.0 & $1.8 \pm 0.6$ \\
\hline \multicolumn{15}{|l|}{ 6- Movement } \\
\hline $\begin{array}{l}\text { How far do you } \\
\text { satisfy your } \\
\text { movement? }\end{array}$ & 2 & 5.0 & 26 & $\begin{array}{c}6 \\
5 . \\
0\end{array}$ & 12 & 30.0 & $2.3 \pm 0.5$ & 4 & $\begin{array}{r}10 \\
.0\end{array}$ & 30 & 75.0 & 6 & 15.0 & $2.1 \pm 0.5$ \\
\hline $\begin{array}{l}\text { How far do you } \\
\text { upset difficulties in } \\
\text { movement? }\end{array}$ & 19 & $\begin{array}{c}47 . \\
5\end{array}$ & 14 & $\begin{array}{l}3 \\
5 . \\
0\end{array}$ & 7 & 17.5 & $1.7 \pm 0.8$ & 7 & $\begin{array}{l}17 \\
.5\end{array}$ & 28 & 70.0 & 5 & 12.5 & $2 \pm 0.6$ \\
\hline $\begin{array}{l}\text { Does movement } \\
\text { difficulty affect your } \\
\text { life style? }\end{array}$ & 14 & $\begin{array}{c}35 . \\
0\end{array}$ & 23 & $\begin{array}{c}5 \\
7 . \\
5 \\
\end{array}$ & 3 & 7.5 & $1.7 \pm 0.6$ & 12 & $\begin{array}{l}30 \\
.0\end{array}$ & 25 & 62.5 & 3 & 7.5 & $1.8 \pm 0.6$ \\
\hline \multicolumn{15}{|c|}{ 7- Dependency on medications } \\
\hline $\begin{array}{l}\text { How far do you } \\
\text { depend on } \\
\text { medicines? }\end{array}$ & 12 & $\begin{array}{c}30 . \\
0\end{array}$ & 24 & $\begin{array}{c}6 \\
0 . \\
0\end{array}$ & 4 & 10.0 & $1.8 \pm 0.6$ & 18 & $\begin{array}{r}45 \\
.0\end{array}$ & 20 & 50.0 & 2 & 5.0 & $1.6 \pm 0.6$ \\
\hline $\begin{array}{l}\text { How far do you need } \\
\text { medicines in doing } \\
\text { your daily activities? }\end{array}$ & 13 & $\begin{array}{c}32 . \\
5\end{array}$ & 18 & $\begin{array}{c}4 \\
5 . \\
0\end{array}$ & 9 & 22.5 & $1.9 \pm 0.7$ & 17 & $\begin{array}{r}42 \\
.5\end{array}$ & 20 & 50.0 & 3 & 7.5 & $1.7 \pm 0.6$ \\
\hline $\begin{array}{l}\text { How far does your } \\
\text { life quality depend } \\
\text { on medicines? }\end{array}$ & 12 & $\begin{array}{c}30 . \\
0\end{array}$ & 22 & $\begin{array}{l}5 \\
5 . \\
0\end{array}$ & 6 & 15.0 & $1.9 \pm 0.7$ & 19 & $\begin{array}{r}47 \\
.5\end{array}$ & 18 & 45.0 & 3 & 7.5 & $1.6 \pm 0.6$ \\
\hline
\end{tabular}

Table (3): Distribution of the studied sample as regards to psychological domain $(n=80)$.

\begin{tabular}{|c|c|c|c|c|c|c|c|c|c|c|c|c|c|c|}
\hline \multirow{3}{*}{$\begin{array}{l}\text { Psychologi } \\
\text { cal domain }\end{array}$} & \multicolumn{7}{|c|}{ Assiut $(n=40)$} & \multicolumn{7}{|c|}{ Umulj $(n=40)$} \\
\hline & \multicolumn{2}{|c|}{ Low } & \multicolumn{2}{|c|}{ Moderate } & \multicolumn{2}{|c|}{ High } & \multirow{2}{*}{$\begin{array}{l}\text { Mean } \\
\pm \text { SD } \\
\end{array}$} & \multicolumn{2}{|c|}{ Low } & \multicolumn{2}{|c|}{ Moderate } & \multicolumn{2}{|c|}{ High } & \multirow{2}{*}{$\begin{array}{l}\text { Mean } \\
\pm \text { SD }\end{array}$} \\
\hline & No & $\%$ & No & $\%$ & No & $\%$ & & $\mathbf{N}$ & $\%$ & No & $\%$ & No & $\%$ & \\
\hline \multicolumn{15}{|c|}{ 1- Negative feelings } \\
\hline $\begin{array}{l}\text { Do you } \\
\text { feel quick- } \\
\text { Tempered, } \\
\text { desperate } \\
\text { and have } \\
\text { anxious? }\end{array}$ & 9 & 22.5 & 28 & 70.0 & 3 & 7.5 & $1.9 \pm 0.5$ & 16 & 40.0 & 12 & 30.0 & 12 & 30.0 & $1.9 \pm 0.8$ \\
\hline $\begin{array}{l}\text { How far } \\
\text { does your } \\
\text { sadness } \\
\text { prevent } \\
\text { you from } \\
\text { daily } \\
\text { activities? }\end{array}$ & 21 & 52.5 & 15 & 37.5 & 4 & 10.0 & $1.6 \pm 0.7$ & 13 & 32.5 & 21 & 52.5 & 6 & 15.0 & $1.8 \pm 0.7$ \\
\hline $\begin{array}{l}\text { Do you } \\
\text { upset } \\
\text { depression } \\
\text { ? }\end{array}$ & 17 & 42.5 & 13 & 32.5 & 10 & 25.0 & $1.8 \pm 0.8$ & 11 & 27.5 & 21 & 52.5 & 8 & 20.0 & $1.9 \pm 0.7$ \\
\hline
\end{tabular}




\begin{tabular}{|c|c|c|c|c|c|c|c|c|c|c|c|c|c|c|}
\hline \multirow{3}{*}{$\begin{array}{l}\text { Psychologi } \\
\text { cal domain }\end{array}$} & \multicolumn{7}{|c|}{ Assiut $(n=40)$} & \multicolumn{7}{|c|}{ Umuli ( $n=40)$} \\
\hline & \multicolumn{2}{|c|}{ Low } & \multicolumn{2}{|c|}{ Moderate } & \multicolumn{2}{|c|}{ High } & \multirow{2}{*}{$\begin{array}{l}\text { Mean } \\
\pm \text { SD }\end{array}$} & \multicolumn{2}{|c|}{ Low } & \multicolumn{2}{|c|}{ Moderate } & \multicolumn{2}{|c|}{ High } & \multirow{2}{*}{$\begin{array}{l}\text { Mean } \\
\pm \text { SD }\end{array}$} \\
\hline & No & $\%$ & No & $\%$ & No & $\%$ & & $\mathbf{N}$ & $\%$ & No & $\%$ & No & $\%$ & \\
\hline \multicolumn{15}{|l|}{$\begin{array}{l}\text { 2-Positive } \\
\text { feelings }\end{array}$} \\
\hline $\begin{array}{l}\text { Do you } \\
\text { satisfy and } \\
\text { enjoy life? }\end{array}$ & 20 & 50.0 & 12 & 30.0 & 8 & 20.0 & $1.7 \pm 0.8$ & 6 & 15.0 & 26 & 65.0 & 8 & 20.0 & $2.1 \pm 0.6$ \\
\hline $\begin{array}{l}\text { Do you } \\
\text { feel } \\
\text { optimistic } \\
\text { about } \\
\text { future? }\end{array}$ & 6 & 15.0 & 21 & 52.5 & 13 & 32.5 & $2.2 \pm 0.7$ & 2 & 5.0 & 21 & 52.5 & 17 & 42.5 & $2.4 \pm 0.6$ \\
\hline $\begin{array}{l}\text { How do } \\
\text { you feel } \\
\text { sharing in } \\
\text { your life? }\end{array}$ & 12 & 30.0 & 15 & 37.5 & 13 & 32.5 & $2 \pm 0.8$ & 5 & 12.5 & 26 & 65.0 & 9 & 22.5 & $2.1 \pm 0.6$ \\
\hline \multicolumn{15}{|c|}{ 3- Self esteem } \\
\hline $\begin{array}{l}\text { Do you } \\
\text { trust } \\
\text { yourself? }\end{array}$ & 1 & 2.5 & 24 & 60.0 & 15 & 37.5 & $2.4 \pm 0.5$ & 2 & 5.0 & 18 & 45.0 & 20 & 50.0 & $2.5 \pm 0.6$ \\
\hline $\begin{array}{l}\text { How far } \\
\text { do you } \\
\text { satisfy } \\
\text { your } \\
\text { capabilitie } \\
\text { s? }\end{array}$ & 7 & 17.5 & 22 & 55.0 & 11 & 27.5 & $2.1 \pm 0.7$ & 4 & 10.0 & 30 & 75.0 & 6 & 15.0 & $2.1 \pm 0.5$ \\
\hline $\begin{array}{l}\text { How do } \\
\text { you } \\
\text { evaluate } \\
\text { yourself? }\end{array}$ & 6 & 15.0 & 21 & 52.5 & 13 & 32.5 & $2.2 \pm 0.7$ & 0 & 0.0 & 35 & 87.5 & 5 & 12.5 & $2.1 \pm 0.3$ \\
\hline \multicolumn{15}{|c|}{ 4- Personal beliefs } \\
\hline $\begin{array}{l}\text { Do } \\
\text { personal } \\
\text { beliefs } \\
\text { give a } \\
\text { meaning } \\
\text { to your } \\
\text { life? }\end{array}$ & 4 & 10.0 & 26 & 65.0 & 10 & 25.0 & $2.2 \pm 0.6$ & 5 & 12.5 & 29 & 72.5 & 6 & 15.0 & $2 \pm 0.5$ \\
\hline $\begin{array}{lr}\text { How } & \text { far } \\
\text { do } & \text { you } \\
\text { think } & \text { your } \\
\text { life r is } & \text { important? }\end{array}$ & 8 & 20.0 & 17 & 42.5 & 15 & 37.5 & $2.2 \pm 0.7$ & 2 & 5.0 & 26 & 65.0 & 12 & 30.0 & $2.3 \pm 0.5$ \\
\hline $\begin{array}{l}\text { How far } \\
\text { do } \\
\text { personal } \\
\text { beliefs } \\
\text { give you } \\
\text { power to } \\
\text { face }\end{array}$ & 5 & 12.5 & 24 & 60.0 & 11 & 27.5 & $2.2 \pm 0.6$ & 1 & 2.5 & 26 & 65.0 & 13 & 32.5 & $2.3 \pm 0.5$ \\
\hline
\end{tabular}




\begin{tabular}{|c|c|c|c|c|c|c|c|c|c|c|c|c|c|c|}
\hline \multirow{3}{*}{$\begin{array}{l}\text { Psychologi } \\
\text { cal domain }\end{array}$} & \multicolumn{7}{|c|}{ Assiut $(n=40)$} & \multicolumn{7}{|c|}{ Umulj (n=40) } \\
\hline & \multicolumn{2}{|c|}{ Low } & \multicolumn{2}{|c|}{ Moderate } & \multicolumn{2}{|c|}{ High } & \multirow{2}{*}{$\begin{array}{l}\text { Mean } \\
\pm \text { SD }\end{array}$} & \multicolumn{2}{|c|}{ Low } & \multicolumn{2}{|c|}{ Moderate } & \multicolumn{2}{|c|}{ High } & \multirow{2}{*}{$\begin{array}{l}\text { Mean } \\
\pm \text { SD } \\
\end{array}$} \\
\hline & No & $\%$ & No & $\%$ & No & $\%$ & & $\mathbf{N}$ & $\%$ & No & $\%$ & No & $\%$ & \\
\hline $\begin{array}{l}\text { difficultie } \\
\text { s? }\end{array}$ & & & & & & & & & & & & & & \\
\hline
\end{tabular}

Table (4): Distribution of the studied sample as regards to social domain $(n=80)$.

\begin{tabular}{|c|c|c|c|c|c|c|c|c|c|c|c|c|c|c|}
\hline \multirow{3}{*}{ Social domain } & \multicolumn{7}{|c|}{$\begin{array}{l}\text { Assiut } \\
(n=40)\end{array}$} & \multicolumn{7}{|c|}{$\begin{array}{l}\text { Umulj } \\
(n=40)\end{array}$} \\
\hline & \multicolumn{2}{|c|}{ Low } & \multicolumn{2}{|c|}{$\begin{array}{c}\text { Moderat } \\
\text { e }\end{array}$} & \multicolumn{2}{|c|}{ High } & \multirow[b]{2}{*}{$\begin{array}{l}\text { Mean } \\
\pm \text { SD }\end{array}$} & \multicolumn{2}{|c|}{ Low } & \multicolumn{2}{|c|}{$\begin{array}{c}\text { Moderat } \\
\text { e }\end{array}$} & \multicolumn{2}{|c|}{ High } & \multirow[b]{2}{*}{$\underset{\text { SD }}{\text { Mean } \pm}$} \\
\hline & $\begin{array}{l}\text { N } \\
\text { o. }\end{array}$ & $\%$ & $\begin{array}{l}\mathbf{N} \\
\text { o. }\end{array}$ & $\%$ & $\begin{array}{l}\text { N } \\
\text { o. }\end{array}$ & $\%$ & & $\begin{array}{l}\mathbf{N} \\
\mathbf{o}\end{array}$ & $\%$ & $\begin{array}{c}\text { No } \\
\text {. }\end{array}$ & $\%$ & $\begin{array}{l}\mathbf{N} \\
\mathbf{o}\end{array}$ & $\%$ & \\
\hline \multicolumn{15}{|l|}{$\begin{array}{l}\text { 1-Personal } \\
\text { relationship: }\end{array}$} \\
\hline $\begin{array}{l}\text { - How far do you } \\
\text { feel alone in life? }\end{array}$ & $\begin{array}{l}2 \\
0 \\
\end{array}$ & $\begin{array}{c}50 . \\
0\end{array}$ & $\begin{array}{l}1 \\
7 \\
\end{array}$ & 42.5 & 3 & 7.5 & $\begin{array}{c}1.6 \pm 0 \\
.6\end{array}$ & 10 & $\begin{array}{c}25 . \\
0\end{array}$ & 12 & $\begin{array}{c}30 . \\
0\end{array}$ & $\begin{array}{l}1 \\
8 \\
\end{array}$ & 45.0 & $2.2 \pm 0.8$ \\
\hline $\begin{array}{l}\text { - Do you feel } \\
\text { happy with your } \\
\text { family? }\end{array}$ & 5 & $\begin{array}{c}12 . \\
5\end{array}$ & $\begin{array}{l}1 \\
7\end{array}$ & 42.5 & $\begin{array}{l}1 \\
8\end{array}$ & 45.0 & $\frac{2.3 \pm 0}{.7}$ & 1 & 2.5 & 17 & $\begin{array}{c}42 . \\
5\end{array}$ & $\begin{array}{l}2 \\
2\end{array}$ & 55.0 & $2.5 \pm 0.6$ \\
\hline $\begin{array}{l}\text { - How far do you } \\
\text { satisfy your } \\
\text { personal } \\
\text { relationships? }\end{array}$ & 1 & 2.5 & $\begin{array}{l}2 \\
0\end{array}$ & 50.0 & $\begin{array}{l}1 \\
9\end{array}$ & 47.5 & $\begin{array}{l}2.5 \pm 0 \\
.6\end{array}$ & 6 & $\begin{array}{c}15 . \\
0\end{array}$ & 22 & $\begin{array}{c}55 . \\
0\end{array}$ & $\begin{array}{l}1 \\
2\end{array}$ & 30.0 & $2.2 \pm 0.7$ \\
\hline \multicolumn{15}{|l|}{$\begin{array}{l}\text { 2- Help social } \\
\text { support }\end{array}$} \\
\hline $\begin{array}{l}\text { - How far can you } \\
\text { depend on your } \\
\text { friends in need? }\end{array}$ & $\begin{array}{l}1 \\
1\end{array}$ & $\begin{array}{c}27 . \\
5\end{array}$ & $\begin{array}{l}1 \\
9\end{array}$ & 47.5 & $\begin{array}{l}1 \\
0\end{array}$ & 25.0 & $2 \pm 0.7$ & 10 & $\begin{array}{c}25 . \\
0\end{array}$ & 23 & $\begin{array}{c}57 . \\
5\end{array}$ & 7 & 17.5 & $1.9 \pm 0.7$ \\
\hline $\begin{array}{l}\text { - Do you satisfy } \\
\text { the help which you } \\
\text { get from family? }\end{array}$ & 4 & $\begin{array}{c}10 . \\
0\end{array}$ & $\begin{array}{l}1 \\
6\end{array}$ & 40.0 & $\begin{array}{l}2 \\
0\end{array}$ & 50.0 & $\begin{array}{l}2.4 \pm 0 \\
.7\end{array}$ & 5 & $\begin{array}{c}12 \\
5\end{array}$ & 23 & $\begin{array}{c}57 . \\
5\end{array}$ & $\begin{array}{l}1 \\
2\end{array}$ & 30.0 & $2.2 \pm 0.6$ \\
\hline $\begin{array}{l}\text { - How far do you } \\
\text { satisfy your friends } \\
\text { 'help? }\end{array}$ & $\begin{array}{l}1 \\
1\end{array}$ & $\begin{array}{c}27 . \\
5\end{array}$ & $\begin{array}{l}1 \\
0\end{array}$ & 25.0 & $\begin{array}{l}1 \\
9\end{array}$ & 47.5 & $\begin{array}{l}2.2 \pm 0 \\
.9\end{array}$ & 9 & 22 & 25 & $\begin{array}{c}62 . \\
5\end{array}$ & 6 & 15.0 & $1.9 \pm 0.6$ \\
\hline \multicolumn{15}{|l|}{ 3- Sexual activity } \\
\hline $\begin{array}{l}\text { - How do you } \\
\text { evaluate your life } \\
\text { quality? }\end{array}$ & 9 & $\begin{array}{c}22 . \\
5\end{array}$ & $\begin{array}{l}2 \\
5\end{array}$ & 62.5 & 1 & 2.5 & $\begin{array}{l}1.6 \pm 0 \\
.7\end{array}$ & 6 & $\begin{array}{c}15 . \\
0\end{array}$ & 32 & $\begin{array}{c}80 . \\
0\end{array}$ & 2 & 5.0 & $1.9 \pm 0.4$ \\
\hline $\begin{array}{l}\text { - How far do you } \\
\text { satisfy your sexual } \\
\text { life? }\end{array}$ & $\begin{array}{l}1 \\
7\end{array}$ & $\begin{array}{c}42 . \\
5\end{array}$ & $\begin{array}{l}1 \\
6\end{array}$ & 40.0 & 2 & 5.0 & $\frac{1.4 \pm 0}{.8}$ & 16 & $\begin{array}{c}40 . \\
0\end{array}$ & 21 & $\begin{array}{c}52 . \\
5\end{array}$ & 3 & 7.5 & $1.7 \pm 0.6$ \\
\hline $\begin{array}{l}\text { - How far do you } \\
\text { upset your sexual } \\
\text { life? }\end{array}$ & 3 & 7.5 & $\begin{array}{l}1 \\
8\end{array}$ & 45.0 & $\begin{array}{l}1 \\
4\end{array}$ & 35.0 & $2 \pm 1$ & 2 & 5.0 & 24 & $\begin{array}{c}60 . \\
0\end{array}$ & $\begin{array}{l}1 \\
4\end{array}$ & 35.0 & $2.3 \pm 0.6$ \\
\hline
\end{tabular}


Table (5): Distribution of the studied sample as regards to environmental domain $(\mathrm{n}=80)$.

\begin{tabular}{|c|c|c|c|c|c|c|c|c|c|c|c|c|c|c|}
\hline \multirow{3}{*}{$\begin{array}{l}\text { Environmenta } \\
\text { I domain }\end{array}$} & \multicolumn{7}{|c|}{$\begin{array}{l}\text { Assiut } \\
(\mathrm{n}=40)\end{array}$} & \multicolumn{7}{|c|}{$\begin{array}{l}\text { Umulj } \\
(n=40)\end{array}$} \\
\hline & \multicolumn{2}{|c|}{ Low } & \multicolumn{2}{|c|}{$\begin{array}{c}\text { Moderat } \\
\text { e }\end{array}$} & \multicolumn{2}{|c|}{ High } & \multirow{2}{*}{$\underset{D}{\operatorname{Mean} \pm S}$} & \multicolumn{2}{|c|}{ Low } & \multicolumn{2}{|c|}{$\begin{array}{c}\text { Moderat } \\
\text { e }\end{array}$} & \multicolumn{2}{|c|}{ High } & \multirow{2}{*}{$\begin{array}{l}\text { Mean } \\
\pm \text { SD }\end{array}$} \\
\hline & $\begin{array}{c}\text { No } \\
.\end{array}$ & $\%$ & $\begin{array}{c}\text { No } \\
\text {. }\end{array}$ & $\%$ & $\begin{array}{c}\text { No } \\
\text {. }\end{array}$ & $\%$ & & $\begin{array}{c}\text { No } \\
\text {. }\end{array}$ & $\%$ & $\begin{array}{c}\text { No } \\
\text {. }\end{array}$ & $\%$ & $\begin{array}{c}\text { No } \\
\text {. }\end{array}$ & $\%$ & \\
\hline \multicolumn{15}{|c|}{ 1- The surrounding environment (Pollution and Noise): } \\
\hline $\begin{array}{l}\text { - How healthy } \\
\text { is the } \\
\text { surrounding } \\
\text { environment } \\
\text { around you? }\end{array}$ & 5 & $\begin{array}{c}12 \\
5\end{array}$ & 24 & $\begin{array}{c}60 . \\
0\end{array}$ & 11 & $\begin{array}{c}27 . \\
5\end{array}$ & $2.2 \pm 0.6$ & 3 & 7.5 & 35 & $\begin{array}{c}87 . \\
5\end{array}$ & 2 & 5.0 & $2 \pm 0.4$ \\
\hline $\begin{array}{l}\text { - Does the } \\
\text { surrounding } \\
\text { noise upset } \\
\text { you? }\end{array}$ & 14 & $\begin{array}{c}35 . \\
0\end{array}$ & 20 & $\begin{array}{c}50 . \\
0\end{array}$ & 6 & $\begin{array}{c}15 . \\
0\end{array}$ & $1.8 \pm 0.7$ & 8 & $\begin{array}{c}20 . \\
0\end{array}$ & 25 & $\begin{array}{c}62 \\
5\end{array}$ & 7 & $\begin{array}{c}17 . \\
5\end{array}$ & $2 \pm 0.6$ \\
\hline $\begin{array}{l}\text { - Do you } \\
\text { satisfy } \\
\text { climate? }\end{array}$ & 8 & $\begin{array}{c}20 . \\
0\end{array}$ & 17 & $\begin{array}{c}42 . \\
5\end{array}$ & 15 & $\begin{array}{c}37 . \\
5\end{array}$ & $2.2 \pm 0.7$ & 5 & $\begin{array}{c}12 . \\
5\end{array}$ & 34 & $\begin{array}{c}85 . \\
0\end{array}$ & 1 & 2.5 & $\frac{1.9 \pm 0 .}{4}$ \\
\hline \multicolumn{15}{|c|}{ 2- Safety and security: } \\
\hline $\begin{array}{l}\text { - How far do } \\
\text { you feel safe in } \\
\text { daily life? }\end{array}$ & 0 & 0.0 & 28 & $\begin{array}{c}70 . \\
0\end{array}$ & 12 & $\begin{array}{c}30 . \\
0\end{array}$ & $2.3 \pm 0.5$ & 1 & 2.5 & 21 & $\begin{array}{c}52 . \\
5\end{array}$ & $\begin{array}{l}1 \\
8\end{array}$ & $\begin{array}{c}45 . \\
0\end{array}$ & $2.4 \pm 0$ \\
\hline $\begin{array}{l}\text { - Do you feel } \\
\text { as you live in a } \\
\text { safe and secure } \\
\text { environment? }\end{array}$ & 4 & $\begin{array}{c}10 . \\
0\end{array}$ & 18 & $\begin{array}{c}45 . \\
0\end{array}$ & 18 & $\begin{array}{c}45 . \\
0\end{array}$ & $2.4 \pm 0.7$ & 2 & 5.0 & 18 & $\begin{array}{c}45 . \\
0\end{array}$ & $\begin{array}{l}2 \\
0\end{array}$ & $\begin{array}{c}50 . \\
0\end{array}$ & $2.5 \pm 0$. \\
\hline $\begin{array}{l}\text { - How far do } \\
\text { you worry } \\
\text { about your } \\
\text { safety and } \\
\text { security? }\end{array}$ & 19 & $\begin{array}{c}47 . \\
5\end{array}$ & 16 & $\begin{array}{c}40 . \\
0\end{array}$ & 5 & $\begin{array}{c}12 \\
5\end{array}$ & $1.7 \pm 0.7$ & 10 & $\begin{array}{c}25 . \\
0\end{array}$ & 21 & $\begin{array}{c}52 . \\
5\end{array}$ & 9 & 22. & $2 \pm 0.7$ \\
\hline \multicolumn{15}{|c|}{ 3- Medical care and social services: } \\
\hline $\begin{array}{l}\text { - How far do } \\
\text { you easily get } \\
\text { a good medical } \\
\text { care? }\end{array}$ & 3 & 7.5 & 25 & $\begin{array}{c}62 \\
5\end{array}$ & 12 & $\begin{array}{c}30 . \\
0\end{array}$ & $2.2 \pm 0.6$ & 0 & 0.0 & 23 & $\begin{array}{c}57 . \\
5\end{array}$ & $\begin{array}{l}1 \\
7\end{array}$ & $\begin{array}{c}42 . \\
5\end{array}$ & $\frac{2.4 \pm 0}{5}$ \\
\hline $\begin{array}{l}\text { - How do you } \\
\text { evaluate the } \\
\text { available social } \\
\text { services } \\
\text { quality? }\end{array}$ & 3 & 7.5 & 19 & $\begin{array}{c}47 . \\
5\end{array}$ & 18 & $\begin{array}{c}45 . \\
0\end{array}$ & $2.4 \pm 0.6$ & 1 & 2.5 & 23 & $\begin{array}{c}57 . \\
5\end{array}$ & $\begin{array}{l}1 \\
6\end{array}$ & $\begin{array}{c}40 . \\
0\end{array}$ & $2.4 \pm 0$ \\
\hline $\begin{array}{l}\text { - How far do } \\
\text { you satisfy } \\
\text { getting medical } \\
\text { care? }\end{array}$ & 10 & $\begin{array}{c}25 . \\
0\end{array}$ & 13 & $\begin{array}{c}32 . \\
5\end{array}$ & 17 & $\begin{array}{c}42 . \\
5\end{array}$ & $2.2 \pm 0.8$ & 1 & 2.5 & 22 & $\begin{array}{c}55 . \\
0\end{array}$ & $\begin{array}{l}1 \\
7\end{array}$ & $\begin{array}{c}42 . \\
5\end{array}$ & $\frac{2.4 \pm 0}{5}$ \\
\hline
\end{tabular}


Table (6): Distribution of the studied sample according to perceived quality of life in Assiut and Umulj group $(\mathbf{n}=\mathbf{8 0})$.

\begin{tabular}{|c|c|c|c|c|c|c|c|c|c|c|c|c|c|c|}
\hline \multirow{3}{*}{$\begin{array}{l}\text { perceive } \\
\text { d quality } \\
\text { of life }\end{array}$} & \multicolumn{7}{|c|}{$\begin{array}{l}\text { Assiut } \\
(n=40)\end{array}$} & \multicolumn{7}{|c|}{$\begin{array}{l}\text { Umulj } \\
(n=40)\end{array}$} \\
\hline & \multicolumn{2}{|c|}{ Low } & \multicolumn{2}{|c|}{ Moderate } & \multicolumn{2}{|c|}{ High } & \multirow{2}{*}{$\underset{\text { D }}{\operatorname{Mean} \pm S}$} & \multicolumn{2}{|c|}{ Low } & \multicolumn{2}{|c|}{ Moderate } & \multicolumn{2}{|c|}{ High } & \multirow{2}{*}{$\underset{\text { D }}{\operatorname{Mean} \pm S}$} \\
\hline & $\begin{array}{c}\text { No } \\
\text {. }\end{array}$ & $\%$ & No & $\%$ & $\begin{array}{c}\text { No } \\
\text {. }\end{array}$ & $\%$ & & No & $\%$ & No & $\%$ & No & $\%$ & \\
\hline $\begin{array}{l}\text { - How do } \\
\text { evaluate } \\
\text { your life? }\end{array}$ & 17 & $\begin{array}{r}42 \\
5\end{array}$ & 9 & $\begin{array}{c}22 . \\
5\end{array}$ & 14 & $\begin{array}{c}35 . \\
0\end{array}$ & $2.2+0.6$ & 4 & $\begin{array}{c}10 . \\
0\end{array}$ & 30 & $\begin{array}{c}75 . \\
0\end{array}$ & 6 & $\begin{array}{c}15 . \\
0\end{array}$ & $2.4+0.5$ \\
\hline $\begin{array}{l}\text { - How far } \\
\text { do you } \\
\text { satisfy } \\
\text { your life } \\
\text { quality? }\end{array}$ & 16 & $\begin{array}{c}40 \\
0\end{array}$ & 10 & $\begin{array}{c}25 . \\
0\end{array}$ & 14 & $\begin{array}{c}35 . \\
0\end{array}$ & $2.4+0.6$ & 10 & $\begin{array}{c}25 . \\
0\end{array}$ & 24 & $\begin{array}{c}60 . \\
0\end{array}$ & 6 & $\begin{array}{c}15 . \\
0\end{array}$ & $2.4+0.5$ \\
\hline $\begin{array}{l}\text { - How far } \\
\text { do you } \\
\text { satisfy } \\
\text { your } \\
\text { health? }\end{array}$ & 17 & $\begin{array}{r}42 \\
5\end{array}$ & 10 & $\begin{array}{c}25 . \\
0\end{array}$ & 13 & $\begin{array}{c}32 . \\
5\end{array}$ & $2.2+0.8$ & 6 & $\begin{array}{c}15 . \\
0\end{array}$ & 31 & $\begin{array}{c}77 . \\
5\end{array}$ & 3 & 7.5 & $2.4+0.5$ \\
\hline
\end{tabular}

Table (7) Distribution of the studied sample according to all domains of quality of life domain in Assiut and Umulj group $(\mathbf{n}=\mathbf{8 0})$.

\begin{tabular}{|l|c|c|c|c|c|}
\hline \multirow{2}{*}{ Variable } & \multicolumn{2}{|c|}{$\begin{array}{c}\text { Assiut } \\
(\mathbf{n}=\mathbf{4 0})\end{array}$} & \multicolumn{2}{c|}{$\begin{array}{l}\text { Umulj } \\
(\mathbf{n}=\mathbf{4 0})\end{array}$} & \multirow{2}{*}{ P. value } \\
\cline { 2 - 5 } & Range & Mean \pm SD & Range & Mean \pm SD & \\
\hline $\begin{array}{l}1 . \quad \text { Physical domain } \\
\text { (score=63) }\end{array}$ & $32-48$ & $39.7 \pm 4.3$ & $32-49$ & $38.8 \pm 4.3$ & 0.337 \\
\hline $\begin{array}{l}\text { 2. psychological domain } \\
\text { (score=36) }\end{array}$ & $19-33$ & $24.3 \pm 3.3$ & $17-34$ & $25.4 \pm 3.8$ & 0.164 \\
\hline $3 . \quad$ Social domain (score=27) & $13-24$ & $17.9 \pm 2.7$ & $13-25$ & $18.8 \pm 3.2$ & 0.178 \\
\hline $\begin{array}{l}\text { 4. Environmental domain } \\
\text { (score=27) }\end{array}$ & $15-23$ & $19.2 \pm 2.1$ & $15-24$ & $19.9 \pm 1.9$ & 0.120 \\
\hline $\begin{array}{l}\text { 5. Perceive quality of life } \\
\text { (score=153) }\end{array}$ & $92-116$ & $101 \pm 7.4$ & $83-123$ & $102.8 \pm 9.7$ & 0.355 \\
\hline
\end{tabular}


Table (8) : Distribution of the studied sample according to quality of life level in Assiut and Umulj group $(n=$ 80).

\begin{tabular}{|c|c|c|c|c|c|}
\hline \multirow{3}{*}{ Quality of life level } & \multicolumn{4}{|c|}{ Country } & \multirow{3}{*}{ P. value } \\
\hline & \multicolumn{2}{|c|}{ Assiut $(n=40)$} & \multicolumn{2}{|c|}{ Umulj $(n=40)$} & \\
\hline & No. & $\%$ & No. & $\%$ & \\
\hline High & 15 & 37.5 & 16 & 40.0 & \multirow{2}{*}{0.500} \\
\hline Moderate & 25 & 62.5 & 24 & 60.0 & \\
\hline
\end{tabular}

Table (9): Relationship between biosciodemographic hemodialysis patient's characteristics and quality of life level in Assiut and Umulj group $(n=80)$.

\begin{tabular}{|c|c|c|c|c|c|c|c|c|c|c|}
\hline \multirow{4}{*}{ Variable } & \multicolumn{10}{|c|}{ Quality of life level } \\
\hline & \multicolumn{5}{|c|}{ Assiut } & \multicolumn{5}{|c|}{ Umulj } \\
\hline & \multicolumn{2}{|c|}{ Moderate $(n=25)$} & \multicolumn{2}{|c|}{ High $(n=15)$} & \multirow{2}{*}{$\begin{array}{c}P . \\
\text { value }\end{array}$} & \multicolumn{2}{|c|}{ Moderate $(n=24)$} & \multicolumn{2}{|c|}{ High $(n=16)$} & \multirow{2}{*}{$\begin{array}{c}\mathbf{P} . \\
\text { value }\end{array}$} \\
\hline & No. & $\%$ & No. & $\%$ & & No. & $\%$ & No. & $\%$ & \\
\hline \multicolumn{11}{|l|}{ Age groups } \\
\hline $18-29$ years & 3 & 12.0 & 3 & 20.0 & \multirow{4}{*}{0.546} & 4 & 16.7 & 5 & 31.3 & \multirow{4}{*}{0.670} \\
\hline $30-39$ years & 7 & 28.0 & 4 & 26.7 & & 1 & 4.2 & 1 & 6.3 & \\
\hline $40-49$ years & 8 & 32.0 & 2 & 13.3 & & 6 & 25.0 & 4 & 25.0 & \\
\hline $50-65$ years & 7 & 28.0 & 6 & 40.0 & & 13 & 54.2 & 6 & 37.5 & \\
\hline \multicolumn{11}{|l|}{ Gender } \\
\hline Male & 18 & 72.0 & 3 & 20.0 & \multirow{2}{*}{$\begin{array}{c}0.003 \\
* *\end{array}$} & 14 & 58.3 & 7 & 43.8 & \multirow[t]{2}{*}{0.520} \\
\hline Female & 7 & 28.0 & 12 & 80.0 & & 10 & 41.7 & 9 & 56.3 & \\
\hline \multicolumn{11}{|l|}{ Marital status } \\
\hline Single & 3 & 12.0 & 2 & 13.3 & \multirow{4}{*}{0.982} & 4 & 16.7 & 5 & 31.3 & \multirow{4}{*}{0.604} \\
\hline Married & 20 & 80.0 & 12 & 80.0 & & 12 & 50.0 & 8 & 50.0 & \\
\hline Divorced & 0 & 0.0 & 0 & 0.0 & & 4 & 16.7 & 1 & 6.3 & \\
\hline Widowed & 2 & 8.0 & 1 & 6.7 & & 4 & 16.7 & 2 & 12.5 & \\
\hline \multicolumn{11}{|l|}{ Education level } \\
\hline Illiterate & 6 & 24.0 & 7 & 46.7 & \multirow{5}{*}{0.105} & 9 & 37.5 & 5 & 31.3 & \multirow{5}{*}{0.305} \\
\hline $\begin{array}{l}\text { Reading and } \\
\text { writing }\end{array}$ & 2 & 8.0 & 3 & 20.0 & & 8 & 33.3 & 2 & 12.5 & \\
\hline Basic education & 3 & 12.0 & 1 & 6.7 & & 5 & 20.8 & 7 & 43.8 & \\
\hline $\begin{array}{l}\text { Secondary } \\
\text { school }\end{array}$ & 13 & 52.0 & 2 & 13.3 & & 0 & 0.0 & 0 & 0.0 & \\
\hline University & 1 & 4.0 & 2 & 13.3 & & 2 & 8.3 & 2 & 12.5 & \\
\hline \multicolumn{11}{|l|}{ Occupation } \\
\hline Not working & 12 & 48.0 & 3 & 20.0 & \multirow{6}{*}{$0.038 *$} & 15 & 62.5 & 4 & 25.0 & \multirow{6}{*}{0.129} \\
\hline Worker & 1 & 4.0 & 1 & 6.7 & & 1 & 4.2 & 2 & 12.5 & \\
\hline Employee & 6 & 24.0 & 0 & 0.0 & & 3 & 12.5 & 3 & 18.8 & \\
\hline Student & 1 & 4.0 & 1 & 6.7 & & 0 & 0.0 & 0 & 0.0 & \\
\hline House wife & 5 & 20.0 & 9 & 60.0 & & 5 & 20.8 & 7 & 43.8 & \\
\hline Others & 0 & 0.0 & 1 & 6.7 & & 0 & 0.0 & 0 & 0.0 & \\
\hline \multicolumn{11}{|l|}{ Residence } \\
\hline City & 9 & 36.0 & 7 & 46.7 & \multirow[t]{2}{*}{0.368} & 20 & 83.3 & 13 & 81.3 & 0.592 \\
\hline Rural & 16 & 64.0 & 8 & 53.3 & & 4 & 16.7 & 3 & 18.8 & \\
\hline
\end{tabular}




\begin{tabular}{|c|c|c|c|c|c|c|c|c|c|c|}
\hline \multirow{4}{*}{ Variable } & \multicolumn{10}{|c|}{ Quality of life level } \\
\hline & \multicolumn{5}{|c|}{ Assiut } & \multicolumn{5}{|c|}{ Umulj } \\
\hline & \multicolumn{2}{|c|}{ Moderate(n=25) } & \multicolumn{2}{|c|}{ High $(n=15)$} & \multirow{2}{*}{$\begin{array}{c}P . \\
\text { value }\end{array}$} & \multicolumn{2}{|c|}{ Moderate(n=24) } & \multicolumn{2}{|c|}{ High $(n=16)$} & \multirow{2}{*}{$\begin{array}{c}\text { P. } \\
\text { value }\end{array}$} \\
\hline & No. & $\%$ & No. & $\%$ & & No. & $\%$ & No. & $\%$ & \\
\hline $1-3$ person & 6 & 24.0 & 1 & 6.7 & \multirow{3}{*}{0.071} & 3 & 12.5 & 3 & 18.8 & \multirow{3}{*}{0.853} \\
\hline $4-6$ person & 14 & 56.0 & 6 & 40.0 & & 7 & 29.2 & 4 & 25.0 & \\
\hline $7 \&$ more & 5 & 20.0 & 8 & 53.3 & & 14 & 58.3 & 9 & 56.3 & \\
\hline \multicolumn{11}{|c|}{ Duration of hemodialysis } \\
\hline 6 months- 1 year & 2 & 8.0 & 2 & 13.3 & \multirow{3}{*}{0.772} & 4 & 16.7 & 3 & 18.8 & \multirow{3}{*}{0.621} \\
\hline 1 years -3 years & 7 & 28.0 & 3 & 20.0 & & 4 & 16.7 & 1 & 6.3 & \\
\hline 3 years $\&$ more & 16 & 64.0 & 10 & 66.7 & & 16 & 66.7 & 12 & 75.0 & \\
\hline \multicolumn{11}{|c|}{ Number of hemodialysis } \\
\hline Two & 1 & 4.0 & 0 & 0.0 & \multirow[b]{2}{*}{0.433} & 0 & 0.0 & 0 & 0.0 & \multirow[t]{2}{*}{$\mathrm{NA}$} \\
\hline Three or more & 24 & 96.0 & 15 & 100.0 & & 24 & 100.0 & 16 & 100.0 & \\
\hline \multicolumn{11}{|c|}{ Number of hours / week of hemodialysis } \\
\hline Four & 25 & 100.0 & 14 & 93.3 & \multirow[t]{2}{*}{0.191} & 7 & 29.2 & 5 & 31.3 & \multirow[t]{2}{*}{0.888} \\
\hline Five & 0 & 0.0 & 1 & 6.7 & & 17 & 70.8 & 11 & 68.8 & \\
\hline
\end{tabular}

Table (1) show that, more than quarter of the studied sample in Assiut university hospital and in Umulj hospital; their age had ranged from 50 - 65 years (32.5\%, 47.5\% respectively), more than half of the studied patients were male $(52.5 \%)$, More than two thirds of them were married $(80.0 \%, 50.0 \%$ respectively), regarding educational level; more than one third in Assiut (37.5\%) were having secondary school education and in Umulj $(35.0 \%)$ were illiterate. As regard occupation in both groups most of them were not working and house wives.

The data reveals that the patients' in Assiut more than half live in rural area $60.0 \%$, while in Umulj more than two third live in city $82.5 \%$. As regard family number half of patient were $4-6$ person in Assiut $50.0 \%$ while in Umulj more than half were 7 \& more person $57.5 \%$.

As regard the duration of hemodialysis two third of patients' in Assiut and more than two third in Umulj were had 3 years \& more. The data illustrate that, majority of patient in Assiut and all of patient in Umulj the number of hemodialysis per week three once or more $(97.5 \%, \& 100.0 \%$ respectively).

Table (2): This table illustrates that according to doing daily activities items in Assiut the most patients were moderate able to do daily activities and upset failure in doing activities $(57.5 \%$ \& $40.0 \%$ respectively). $40.0 \%$ with low feeling difficulty in doing usual activities. While doing daily activities items in Umulj more than two third of patients were with moderate able to do daily activities, feeling difficulty in doing usual activities, and upset failure in doing activities $(77.5 \%, 62.5 \%, \& 62.5 \%$ respectively).

According to pain and discomfort items in Assiut the most patients were $52.5 \%$ with moderate suffer from pains, $70.0 \%$ with high worry about pains or discomfort and $72.5 \%$ with low don't do works when feel pain. While in Umulj most patients were moderate suffer from pains, worry about pains or discomfort, \& don't do works when feel pain (62.5 $\%, 70.0 \%, \& 72.5 \%$ respectively).

According to energy and fatigue items in Assiut the more than half of patients were $67.5 \%$ with moderate have enough energy for daily activities, more than one third $47.5 \%$ with low satisfy/pleased with energy and half of the sample $50.0 \%$ with low upset pain. While in Umulj more than half of the patients were moderate have enough energy for daily activities and upset pain $(60.0 \% \& 52.5 \%$ respectively), and more than two third $80.0 \%$ with moderate satisfy/pleased with energy.

As regards to sleep and rest this table revealed that, in Assiut and Umulj more than two third of patients were moderate sleep well $(77.5 \%$ \& $75.0 \%$ respectively). More than half of sample were moderate suffer from difficulty sleeping $(62.5 \%$ \& $60.0 \%$ respectively) in both countries Assiut and Umulj. As regard worry about sleeping problems in Assiut more than one third of patients $45.0 \%$ were low, while in Umulj more than half of patients 52.5 $\%$ were moderate.

For the capability to work items the most patients were $(47.5 \% \& 52.5 \%$ respectively) with low able to 
work in Assiut and Umulj, (42.5\% \& $70.0 \%$ respectively) with moderate feel capability in doing duties in both countries and half of patients $50.0 \%$ with low satisfy capability to work in Assiut, while in Umulj more than two third $62.5 \%$ were moderate satisfy capability to work.

According to movement items in Assiut and Umulj the most patients were with moderate satisfy movement, upset difficulties in movement and movement difficulty affect life style.

For dependency on medications items in Assiut the most patients were $(60.0 \%, 45.0 \%$, \& $55.0 \%$ respectively) with moderate depend on medicines, need medicines in doing daily activities and life quality depend on medicines. While in Umulj half of patients were moderate depend on medicines and need medicines in doing daily activities (50\%), 47.5 $\%$ with low life quality depend on medicines.

Table (3): This table shows that, according to negative feelings items in Assiut more than two third of patients were $70.0 \%$ with moderate feel quickTempered, desperate and have anxious, (52.5\% \& 42.5 respectively) with low sadness prevent from daily activities and upset depression. In Umulj more than one third of patients were $40.0 \%$ with low feel quick-Tempered, desperate and have anxious, and equally moderate $52.5 \%$ in both sadness prevent from daily activities and upset depression

As regards to positive feelings items in Assiut half of patients were $50.0 \%$ with low satisfy and enjoy life, with moderate feel optimistic about future and feel sharing in life $(52.5 \%$ \& $37.5 \%$ respectively). In Umulj most of patients were moderate satisfy and enjoy life, feel optimistic about future and feel sharing in life $(65.0 \%, 52.5 \%, \& 65.0$ respectively). For self-esteem items in Assiut and Umulj the most patients were with moderate trust himself, satisfy capabilities and evaluate himself. As regards to personal beliefs items the most patients in Assiut and Umulj were moderate personal beliefs give a meaning to life, think life is important and personal beliefs give power to face difficulties.

Table (4): This table stated that according to personal relationship items in Assiut the half of patients were $50.0 \%$ with low feel alone in life, more than one third $42.5 \%$ with moderate feel happy with his family and half of patients $50.0 \%$ with moderate personal satisfy personal relationships. In Umulj most of patients were moderate feel alone in life, feel happy with his family, and personal satisfy personal relationships (30.0\&, $42.5 \%$, \& $55.0 \%$ respectively). As regards to help social support items in Assiut and Umulj the patients were moderate in all items except satisfy friends help $47.5 \%$ with high in Assiut.
According to sexual needs items in Assiut and Umulj the patients were moderate in all items.

Table (5): This table shows that according to physical environmental, safety and security, and medical care and social services the patients were moderate in all items in Assiut and Umulj.

Table (6): this table mentioned that according to perceived quality of life domain low in Assiut while moderate in Umulj.

Table (7): this table illustrated that according to all domains of quality of life near equal in both study groups.

Table (8): This table stated that, less than half of the sample in Assiut and Umulj were high QOL (37.5\% and $40.0 \%$ ) respectively. Also the table mentioned that, more than half of the sample in Assiut and Umulj were moderate QOL $(62.5 \%$ and $60.0 \%)$ respectively.

Table (9): This table shows that in Assiut statistical significant relation between biosciodemographic characteristics and quality of life as regard gender and occupation with $\left(\mathrm{P}\right.$-value $\left.=0.003 * * \& 0.038^{*}\right)$ respectively. While in Umulj no statistical relation between biosciodemographic characteristics and quality of life.

\section{Discussion}

Since hemodialysis is an expensive treatment modality for chronic renal failure patients, it is very essential to assess the outcome of therapy in terms of quality of life (Abraham et al. 2012). The study of health related quality of life requires an approach from several perspectives and an in-depth understanding because diseases such as the ESRD affect the quality of life in various areas (GarciaViniegras \& Rodriguez, 2007).

The present study results revealed that; near half of the studied sample their age has ranged from $50-65$ years in both groups, more than half of them were male, married, illiterate and not working. As regard residence; in Assiut more than half of the sample was living in rural areas while in Umulj hospital almost the entire sample was living in city.

According to Zhang et al., (2007) in his study results; the demographic profiles revealed that majority of the patients were in the age group of 31-50 years and there exists a male predominance. And regarding the socioeconomic status, upper middle class people were mostly affected.

The present study had revealed that one case of the studied sample had dialyzed one time a week while almost the entire studied sample in both hospitals, the number of hemodialysis sessions was 3 times or more a week, regarding session duration (hours); almost all of the studied sample in Assiut was 4 hours while in 
Umulj a little more than half had undergone dialysis session for 4 hours.

In accordance with this study result in a study which was conducted by Albert et al., (2011) had revealed that the mean age was $64 \pm 14$ (SD) years, $62 \%$ of the patients were male, and $93 \%$ dialyzed 3 times per week and only $6 \%$ of the sample had dialyzed for 2 times per week and session duration ranged from 3.5 to 4 hours.

Regarding duration of hemodialysis; the present study had shown that more than half of the studied sample in both study hospital their duration of hemodialysis was 3 years or more.

In accordance with the present study results regarding the duration of hemodialysis in a study which was carried out by G. G. Veronica et al. 2014; in their study the majority of their study sample had ranged from 4 years and over, also Abraham et al., 2012; said that the majority of the population had duration of renal failure about 3 years.

Regarding quality of life domains the present study had shown that; there was no statistically significant differences in both the study groups. Regarding physical domain patients from Assiut University hospital were a little bit advanced, while psychological, environmental and social domains patients from Umulj hospital were a little bit also advanced and this could be related to their economically higher level. Generally more than half of the studied samples in both hospitals were having a moderate score in their quality of life.

In a study on the quality of life of hemodialysis patients which was conducted by S. Abraham and colleague 2012; it was found that the QOL of hemodialysis patients were significantly impaired.

Regarding relation between level of quality of life and patient's gender; the present study revealed that; there was a statistically significant relation between level of quality of life and gender as female patients were highly scored on the QOL than males.

This also was in accordance with the study results of Abraham et al. 2012 who stated that; the male patients reported significantly lower QOL scores in all domains compared to female patients. However, the study done by Sathvik et al. (2008); showed that the QOL of females were less than that of males.

\section{Conclusion}

The study concluded that; there was a slight clinical relevant difference exist between the studied hospitals dialysis centers in multiple quality of life domains. Also there was a statistically significant relation between the studied groups regarding their quality of life and both patient's gender and occupation.

\section{Recommendations}

As most of the ESRD patients were depressed and worrying about their health conditions, providing patient counseling will play an important role in improving the QOL by changing their psychological thinking and leading them towards spirituality by removing their misconceptions about the disease this will increase the positive feelings in the patient, which will increase the spirituality level of the patients thereby their concentration, thinking and learning power will also increase. As a result, patients will be more involved in their self activities without any negative feelings.

\section{References}

1. Albert H., Mazairac, Muriel P., Grooteman, Peter J., Blankestijn, E., Lars Penne, Neelke C., van der Weerd, Claire H., den Hoedt, Marinus A., van den Dorpel, Erik Buskens, Menso J. Nube, Piet M., ter Wee, G., Ardine de Wit, \& Michiel L., Bots (2011): Differences in quality of life of hemodialysis patients between dialysis centers.

2. Braga S., Peixoto S., Gomes I., de Assis Acurcio F., Andrade E., Cherchiglia ML. (2011): Factors associated with health-related quality of life in elderly patients on hemodialysis. Revista de saude publica. 45(6):1127-36.

3. Brunner L., \& Suddarth D., (2010): Textbook of medical - surgical nursing. 8th ed. JB Lippincott comp., Philadelphia, New York, 1238-98.

4. De Santo N., Perna A., El Matri A., De Santo RM, Cirillo M., (2012): Survival Is Not Enough. Journal of Renal Nutrition. 22(1):211219.

5. Garcia, L., \& Calvanese, N., (2007): Perceived quality of life and locus of control in patients on replacement therapy for renal function: Dialysis and kidney transplantation. Annals Metropolitan University, 7(1), 205-222.

6. Germin-Petrovic D., Mesaros-Devcic I., Lesac A., Mandic M., Soldatic M., Vezmar D., (2011): Health-related quality of life in the patients on maintenance hemodialysis: the analysis of demographic and clinical factors. Collegium Antropologicum; 35(3):687-93.

7. Guerra-Guerrerro, V., Camargo Plazas, M., Cameron, B., Santos Salas, A., \& Cofre Gonzalez, C., (2014): Understanding the life experience of people on hemodialysis: Adherence to treatment and quality of life. Nephrology Nursing Journal, 41(3), 289-297, 316. 
8. Kusleikaite N., Bumblyte I., Kuzminskis V., Vaiciuniene R., (2010): The association between health-related quality of life and mortality among hemodialysis patients. Medicina. 46(8):531-7.

9. Abraham S., \& Ramachandran A., (2012): Estimation of Quality of Life in Hemodialysis Patients, 74 (6): 580-583.

10. Sathvik B., Parthasarathi G., Narahari M., Gurudev K., (2008): An assessment of the quality of life in hemodialysis patients using the WHOQOL BREF questionnaire. Indian $\mathrm{J}$ Nephrology; 18:141.

11. Tel H., Tel H., (2011) : Quality of life \& social support in Hemodialysis patients. Pak J Med Sci; 27(1):64-67

12. Turkmen K., Yazici R., Solak Y., Guney I., Altintepe L., Yeksan M., (2011): Health-related quality of life, sleep quality, and depression in peritoneal dialysis and hemodialysis patients. Hemodial Int. 16(2):198-206.

13. Oort, F., (2005) : Using structural equation modeling to detect response shifts and true change. Quality of Life Research, 14(3), 587598.

14. Zhang A., Cheng L., Zhu N., Sun L., Wang T., (2007) : Comparison of quality of life and causes of hospitalization between hemodialysis and peritoneal dialysis patients in China. Health Qual Life Outcomes; p.p 5:49. 